Pascale Bourret, Peter Keating and Alberto Cambrosio, "Regulating Diagnosis in Post-Genomic Medicine: Re-Aligning Clinical Judgment?” Social Science \& Medicine, 73 (2011), 816-824

\title{
Regulating diagnosis in post-genomic medicine: Re-aligning clinical judgment?
}

\begin{abstract}
In recent years, genomic technologies have entered oncology. In particular, so-called tumor signatures are now commercially available for diagnosing breast cancer. These new diagnostic tools have expanded the content and meaning of diagnosis, by adding a distinctive prognostic (will the disease recur?) and predictive (how will the disease react to treatment?) dimension to this activity, and modifying the relations between diagnosis and therapy. In particular, they raise the issue of the locus of clinical judgment and clinical decision-making insofar as they involve a re-alignment of the biological and clinical components of medical activities. Using as a case study a debate over the regulation of tests for genomic signatures, this paper examines how the actors problematize the issues related to the introduction of molecular diagnostics into clinical settings.
\end{abstract}

\section{Introduction}

For Harry Marks (1947-2011), colleague, friend, and source of inspiration

Diagnosis, prognosis and therapy are the classical components of medical practice. Each component has its own history. Christakis (1999) has argued, for instance, that although prognosis gradually disappeared from medical textbooks at the turn of the $20^{\text {th }}$ century, it has recently regained lost ground due, in part, to the development of new medical technologies. Jutel (2009) has pleaded for the development of an entire sociological subspecialty devoted to the study of diagnosis. While a focus on these individual components of medical practice is certainly worthwhile, it conceals their mutual, shifting relations. Despite inconsistencies between these domains (Mol, 2002), they must in the end be reconciled if clinical judgment and clinical decision-making are to be justifiable and justified in Boltanski and Thévenot's (2006) sense of these terms. In addition, their interconnection precludes the possibility of their sequential performance. Diagnosis, for instance, does not necessarily precede and dictate therapy: therapeutic success may confirm a tentative diagnosis (Berg, 1992). More generally, these activities easily bleed into one another, both synchronically and diachronically. This paper will argue that the development of post-genomic platforms further conflates diagnostic, prognostic and therapeutic categories and processes, creating anxieties among health practitioners over clinical jurisdictions. One of their main concerns is that non-clinical, laboratory-based results increasingly tend to dictate, rather than simply contribute to clinical decisions, and by the same token encroach upon what was once the uncontested domain of the clinician, namely clinical decision-making. This situation is nicely captured by the following excerpt from an interview with a leading French cancer clinician who, commenting on the transformation of clinical judgment in recent years, noted: "we often call it [clinical expertise] intuition, but it's a complex mix of things that our training and clinical experience have taught us, and now, suddenly, we are faced with a situation where [genomic tests] tell us the opposite of what we thought. We said 
'white' and the test tells us 'black', or the other way round" (interview, June 2009; our translation).

Post-World War II biomedicine is characterized by the development of biomedical platforms that embody the meteoric rise of medical biology (Keating \& Cambrosio, 2003). Quantifiable, biological variables have become ubiquitous within the practice of medicine, and attempts to bring pathology and biology together within a single space of representation have become commonplace. Post-genomic technologies take a further step in this direction. This does not mean that medicine has been reduced to biology (Keating \& Cambrosio, 2004). Physicians continue to exercise clinical judgment, just as pathologists, full-fledged members of the clinical community, continue to act as intermediaries between the bedside and the laboratory. Genomic platforms, however, bear the threat or promise (depending on one's point of view) of decentering clinical decision-making. Laboratory tests are hardly new, but have heretofore remained largely external to the treating physician's clinical judgment: the laboratory test provided a result, the clinician diagnosed. This is still mainly the case, as de-centering entails more a realignment of the biological and clinical components of medical judgment, than a displacement of the clinician. Realignment, however, can have important consequences such as a shift from an individual to a collective epistemic subject - the multi-disciplinary bio-clinical team-as the locus of clinical decision-making (Bourret, 2005), and the inclusion of clinical judgments within laboratory tests.

Promoters of genomic approaches claim that "many 'diseases' are clinical syndromes, defined observationally, that undoubtedly are made up of a collection of distinct pathogenic states" (Woodcock, 2007, p. 166). On this view, molecular diagnostics gives clinicians and researchers access to "more underlying pathogenic processes, without necessarily providing full mechanistic, explanatory data" (Ibid.). As cancer is often cited as a model in this respect, we focus on breast cancer as an instance of a domain transformed by molecular techniques through the identification of different subtypes of the disease, each requiring a specific therapeutic approach. In particular, following a positive biopsy of a suspicious lump in her breast, a patient does not simply receive a generic diagnosis of breast cancer. Rather, in addition to traditional pathological parameters such as staging (how much the cancer has grown and spread) and grading (the state of the cancer cells), present diagnoses include molecular information concerning the biology of her tumor, such as the presence or absence of hormone receptors. On the clinical research front, molecular oncology provides breast cancer clinicians with gene expression profiles of the tumor, i.e. the expression pattern of a set of genes (a.k.a. the tumor's signature) that allow the oncologist to assign the tumor to a molecular subspecies (Perou et al., 2000) and, most importantly, to determine if the tumor is likely to recur following surgery and to react to chemotherapy. The emerging genomic platform thus works along three different lines: diagnostic (what kind of disease?), prognostic (will the disease recur?), and predictive (will the disease react to treatment?). This distinction between prognosis and prediction is quite recent and can be ascribed to the emergence of molecular technologies. On the new platform diagnosis incorporates prognosis and prediction. While diagnosis, prognosis and therapy were hardly separate in the past (e.g., hormone-positive and hormone-negative breast cancers carry a different prognosis and are treated differently), the conflation of these three activities has reached new heights. It has become difficult to define, in practice, where and with whom diagnosis ends, and prognosis and therapy begin. 
The present article is part of a broader, ongoing research project on emerging laboratory-clinicalcommercial interfaces in cancer genomics. It will focus on a specific episode, namely the debates concerning the creation of a separate regulatory category-so-called IVDMIAs or In Vitro Diagnostic Multivariate Index Assays - for gene-expression profiling tests by the U.S. Food and Drug Administration (FDA). As we will see, one of the stakes in the controversy over IVDMIAs was which tests should count as members of this category. A number of tests with functions ranging from the early detection of cancer to the prediction of the genetic risk of common diseases were potential candidates for membership and thus subjects of debate. Our discussion centers on two breast cancer tests that are prognostic of the future course of the disease and predictive of treatment response. Not only did these tests figure prominently in the debate, but the issues surrounding them shed light on ongoing interrogations about the shifting locus of clinical judgment. Regulatory debates and practices offer a unique vantage point for the analysis of biomedical innovation, one that is not confined to upstream (the conception of new tools) or downstream (their deployment in clinical routines) events but, rather, begins "right in the middle of the game" where one is in a position to capture the mutually constitutive relations between these two stages, and, in particular, the realignments that articulate novel platforms and the entities they generate with prior diagnostic and prognostic practices (Keating \& Cambrosio, 2003, p. 334; Cambrosio et al., 2006).

Regulation per se is not the topic of this paper, nor is the regulatory environment within which debates took place. Moreover, given the complexities of these issues, it is no surprise that discussions surrounding the FDA initiative ranged from the economics of innovation, to ideological statements about the freedom to practice medicine, and to more concrete discussions of specific aspects of the proposed regulation. Despite these ramifications, our analysis focuses on clinical practices and medical judgment. For while policy and economic considerations play a role in clinical activities, the latter cannot be reduced to the former. Finally, we have chosen, as is now commonplace in science and technology studies, to follow the actors and their statements, thereby implementing a "sociology of critical capacity" rather than a critical sociology (Latour, 2005; Boltanski \& Thévenot, 1999). In the present case, the FDA initiative and the arguments deployed by its supporters clearly reflect the belief that genomic tests are likely to have profound consequences on the relations between biology and medicine, displacing the locus of clinical judgment, readjusting the biomedical division of labor, modifying doctor-patient relations and creating new relations between public, not-for-profit, and commercial institutions. Opponents propose a different socio-technical scenario, one that privileges the resilience of existing sociotechnical arrangements. This clash of visions provides us with an opportunity to consider the shifting role and nature of diagnosis within the onslaught of molecular technologies.

\section{Methodology}

As noted above, the present article is part of a broader research project on cancer genomics that began in 2008 and was approved by McGill University's IRB (\#A05-E11-04B and A02-E1209B). It deploys a three-pronged ethnographic approach that consists of: (a) the systematic collection and analysis of published and unpublished documents (scientific articles, company reports, web newsletters and blogs); (b) interviews with cancer clinicians, researchers, statisticians and the staff of biotech companies on both sides of the Atlantic (by March 2011, approximately 30); and (c) participant-observation at scientific meetings and within three clinical 
settings (in the US, Canada and France). As part of this fieldwork we regularly encountered material directly related to the regulation of genomic signatures: for instance, during a molecular pathology meeting a special session was devoted to the FDA initiative, interviewees offered their (sometimes unsolicited) opinion on this topic, and genomic web sites regularly report on regulatory issues. While our fieldwork did not specifically target regulation, the latter emerged as a key preoccupation of actors in the field, and we were thus able to extract related material from our notes and interviews.

Concerning the FDA regulation itself, we have analyzed the 2006 and 2007 versions of the FDA Draft Guidance documents on IVDMIAs (henceforth First Draft Guidance and Second Draft Guidance), the verbatim transcription of a 2007 Public Meeting organized by the FDA (henceforth FDA Meeting), as well as the written briefs submitted by academic, professional, commercial and advocacy organizations, all 173 of which are available online on the Federal Docket Management System (www.regulations.gov, Docket number 2006D-0347; henceforth Dockets followed by the document ID). A 2008 Citizen Petition on in-vitro diagnostics submitted by Genentech also generated a fair amount of discussion, much of it available in Docket 2008-P0638. More recently, the FDA's decision to extend its proposed oversight to all laboratorydeveloped tests (LDTs) led to another public meeting in July 2010 (henceforth LDT Meeting). A webcast and a verbatim transcription of that meeting, as well as related docket documents (FDA2010-N-0274), are also available on the web. Access to this documentation (in particular webcasts) allows social scientists to "virtually witness" discussions that were previously accessible only via direct participation, thus expanding the reach of qualitative analysts.

In line with "pragmatic sociology" (Nachi, 2006) as applied to medical topics (Moreira, 2005; Knaapen et al., 2010) our analysis focuses on the justifications offered by participants in support or against the proposed FDA oversight of genomic tests, rather than attempting, for instance, to correlate participants' statements with a priori interests or their professional position. In addition to the more general arguments supporting this approach (Boltanski \& Thévenot, 1999), in this particular case both opponents and supporters of the proposed regulation are to be found within each social category (manufacturers, patient advocates, etc.). More importantly, the analysis of justifications allows us to investigate evolving distinctions between actors' categories such as analytical and clinical validity that buttress attempts to reorder the relations between the various components of diagnosis, and between diagnosis and therapy.

\section{Genomic tests and the end of FDA's "enforcement discretion"}

\subsection{A short chronology of events}

All key indicators (number of papers published, grants and patents awarded, and the like) show that cancer is the primary domain for the application of genomic technologies. Their deployment has resulted in the definition of new subtypes of this family of diseases (Perou et al., 2000; Alizadeh et al., 2000), and led to the clinical implementation of molecular tests for cancer prognosis and prediction. Although many of these technologies remain experimental, a number of commercial tests have made significant inroads, such as the breast tumor signatures MammaPrint and Oncotype Dx marketed, respectively, by Agendia, a spin-off of the Netherlands Cancer Institute, and the California company Genomic Health (Kohli-Laven et al., 2011). 
Regulatory debates concerning genomics can be traced back to the late 1990s when, in the wake of the Human Genome Project and following recommendations by a joint working group of the National Institutes of Health and the Department of Energy, the Secretary's Advisory Committee on Genetic Testing (subsequently replaced under the Bush Administration by the Secretary's Advisory Committee on Genetics, Health and Society) recommended increased oversight of genetic technologies, broadly construed to include both inherited and somatic mutations. As far as this paper is concerned, however, the real story begins in September of 2006, when the FDA's Office of In Vitro Diagnostic Device Evaluation and Safety issued a document entitled In Vitro Diagnostic Multivariate Index Assays: Draft Guidance for Industry, Clinical Laboratories and FDA Staff. Published concurrently with another Guidance for analyte specific reagents (ASRs), i.e. the specific components of in-house tests, the First Draft guidance did not simply propose a regulatory framework for an existing kind of assay: it defined a new regulatory category and thus, prospectively, a new kind of assay. The FDA initiative quickly created a regulatory policy storm. A journal for manufacturers of in vitro diagnostic products noted that "both laboratories and IVD manufacturers will remember for a long time" the day on which the FDA issued the two documents, for they "may significantly affect not only the laboratories' abilities to develop and offer new tests, but also existing lab-developed tests" (Gibbs, 2007).

The FDA described its initiative as tentative and invited the public to submit comments to help the agency clarify the contours of the new diagnostic domain (FDA Meeting, pp. 8-9). As expected, a polarizing debate ensued. Regardless of their position, many stakeholders noted that the new assay category lacked clearly defined boundaries. Given the buzz generated by the document, the FDA extended the comment period and eventually received approximately 60 sets of comments, whose authors included not only device manufacturers, but also representatives of professional associations, healthcare organizations, patient advocates, laboratory researchers, clinicians, and other health professionals. In February 2007, the FDA organized a public meeting to provide stakeholders with an opportunity to expose their "concerns, issues and ideas": 266 people attended the meeting, 31 of whom spoke for themselves or as representatives of various organizations to the assembled audience (FDA Meeting, p. 4). Following the public meeting, the FDA issued a second Draft guidance in July of 2007, followed by a new comment period.

While the new document incorporated remarks, suggestions and objections made during the public consultation process, it did not appease all critics. Opponents of the new regulation intensified their activities and mobilized coalitions such as the Genetic Alliance, the "world's leading nonprofit health advocacy organization committed to transforming health through genetics ... [whose] network includes more than 1,000 disease-specific advocacy organizations, as well as thousands of universities, private companies, government agencies, and public policy organizations" (http://geneticalliance.org), and the Coalition for $21^{\text {st }}$ Century Medicine (http://www.twentyfirstcenturymedicine.org), established in 2006 in response to the aforementioned FDA initiatives. Supporters of an increased FDA oversight of genomic tests regrouped under a competing network launched in 2004, the Personalized Medicine Coalition (http://www.personalizedmedicinecoalition.org).

In February of 2007, in parallel with the public meeting, the FDA cleared its first IVDMIA, Agendia's MammaPrint. That same year Agendia joined the Personalized Medicine Coalition and, in 2008, opened a US branch in California. In spite of letters in 2005 and 2006 from the 
FDA suggesting that it "was considering whether Oncotype DX may be subject to FDA premarket review" (Lakhman, 2006), Agendia's main competitor, Genomic Health (a member of the Genetic Alliance and of the Coalition for $21^{\text {st }}$ Century Medicine networks), decided not to submit its test to the FDA, arguing that the agency had not yet issued a final, legally binding Guidance. Instead, the firm opted for professional recognition via inclusion of the test in the breast cancer guidelines issued by two leading US cancer organizations, the National Comprehensive Cancer Network and the American Society of Clinical Oncology. Partly as a reaction to the Genomic Health strategy, in 2008 Genentech submitted a Citizen Petition to the FDA requesting that all laboratory-developed tests (LDTs), of which IVDMIAs are a subset, be subject to FDA scrutiny.

Following the 2009 transition from the Bush to the Obama Administration, and the consequent change in FDA leadership, rather than issuing a final IVDMIA guidance document, the Agency decided to pursue the route advocated in the Citizen Petition by expanding its proposed oversight to all LDTs. The change from an administration wary of state regulations to one more favorable certainly looms large as a cause of this decision, but while the transfer of power may have acted as a condition of possibility for the change, it clearly did not dictate the specific content of regulatory initiatives. The request to broaden the scope of regulations from IVDMIA to other LDTs had emerged from within the debates about IVDMIAs. It was the result of a dynamic process of tinkering and reshuffling the categories and arguments that framed the debate, rather than the mechanical outcome of socio-political determinants. Moreover, the FDA, who had been following developments in pharmacogenomics for a number of years, had meanwhile been confronted with the "incredible rate of growth for genomics and biomarkers in regulatory submissions” (Q\&A: FDA's Zineh..., 2010). But it was more than a question of quantity. While traditional IVDs are commonly used for diagnosing and monitoring patients, the new tests are used to make therapeutic decisions, and as such they were held by a number of participants in the IVDMIA controversy to require clinical validation, as is the case for drugs. The FDA thus began to worry that by formatting their products as LDTs, manufacturers sought to avoid regulations based on the scientific and clinical validity of claims. In the meantime, IVDMIA, as a hybrid scientific-regulatory category, has made some inroads, and now has a dedicated website (http://www.ivdmia.com/).

\subsection{Why IVDMIAs}

Why did the FDA deem it necessary to introduce a novel regulatory category? To answer this question we need to quickly review the existing regulation of diagnostics, which makes provision for two kinds of tools. The first, medical devices, is the category under which the FDA regulates in vitro diagnostic kits (IVDs), i.e. commercial products used by health professionals and consumers to detect diseases and other conditions. IVDs circulate outside their laboratory of origin. Since 1976, using a risk-based approach, medical devices have been subdivided into three classes according to the degree of control necessary to ensure their safety and efficacy. Low-risk Class I devices are mostly exempt from pre-market submissions. Manufacturers of moderate risk, class II devices can avail themselves of a relatively light regulatory procedure known as Premarket Notification or $510(\mathrm{k})$. High-risk, Class III devices require a far more expensive and time-consuming Premarket Approval Application (PMA). A second category of tests consists of laboratory-developed tests (LDTs), nicknamed home brews, i.e. tests designed by and performed 
in diagnostic laboratories. Although the FDA has the power to regulate the safety and effectiveness of home brews, it has traditionally declined to do so, exercising its enforcement discretion (Evans 2006, p. 768). Thus, for all practical purposes LDTs fall under the regulatory authority of the Centers for Medicare and Medicaid Services through the Clinical Laboratory Improvement Amendments (CLIA). This means that the laboratory will be inspected and certified to make sure that it has implemented the necessary quality control and quality assessment procedures, and that the home brew test has analytical validity, i.e. that it measures accurately and consistently whatever it is supposed to measure. LDTs, contrary to kits, do not circulate and the FDA has thus considered them laboratory services rather than medical devices. In this set up, CLIA oversees laboratory services, not the tests, and it does not require data on a test's clinical (as opposed to analytical) validity, i.e. the accuracy with which a test predicts a clinical disorder or outcome.

While assays like the aforementioned Oncotype DX and MammaPrint do not embody all possible diagnostic instantiations of genomics, they have acquired an emblematic status in the FDA IVDMIA debate; they bear many of the conundrums raised by recent diagnostic developments and, most interestingly, representatives of the two companies sat in opposing camps vis-à-vis the FDA initiative. The two tests have been developed in the companies' laboratories and are performed in-house. Clinicians send tumor samples to the Agendia and Genomic Health CLIAcertified laboratories that, in turn, do the analysis and return the results to the clinician. In the case of Oncotype DX, the result is expressed as a Recurrence Score (RS) between 0 and 100 that corresponds to the likelihood of breast cancer recurrence within 10 years of initial diagnosis. MammaPrint's result is expressed as a dichotomy: the profile generated by a patient's tumor is assigned to either a good or poor signature category, corresponding to the likelihood that the patient will relapse. Thus, while both tests use algorithms to calculate the outcome on the basis of the measured gene expression, the Agendia test provides the clinician with a qualitative verdict: the patient is high-risk and should be prescribed chemotherapy, or the patient is low-risk and can avoid it. Genomic Health's RS offers a numerical response on a continuous scale, although in its practical implementation the test also leads to a qualitative verdict, insofar as the RS continuum is divided into three categories: low, intermediate and high risk; low and possibly also intermediate-risk patients can avoid chemotherapy. Notice the links between prognosis and therapy, and the presence of algorithms to generate the result that is transmitted to the clinician. The statistical analyses at the core of devices such as Oncotype DX and MammaPrint are not simply a matter of calibrating and adjusting the measurement of target substances. Rather, they are used to predict specific health outcomes and thus raise the issue of the clinical validity of the tumor signature they generate.

In the eyes of the FDA, these characteristics - in particular the fact that "basically an IVDMIA is a test that is developed using clinical data" (FDA Meeting, p. 7) - clearly separate the new tests from traditional tests. More than a mere discrepancy, in the agency's opinion IVDMIAs play havoc with the categories and parameters that underlie the regulation of diagnostics. The circulation criterion - i.e., whether a test remains confined to a laboratory or circulates as a kitis no longer relevant. Thus, CLIA-regulated laboratories that develop and perform IVDMIAs should be considered as "manufacturers of medical devices" even though these activities occur in a single laboratory. In his introductory comments to the public (FDA Meeting, p. 6), an FDA official provided the following rationale for the agency's initiative: "we have noticed a growing category of tests that include elements that are not standard primary ingredients of laboratory 
developed tests, and we believe that these differences, such as complex, statistically driven data derived algorithms actually raise new safety and efficacy concerns". An IVDMIA, in other words, cannot be reduced to a specific combination of reagents for it includes an algorithm that is derived from clinical studies to which users of the test are not privy and without which the test could not have been validated and, arguably, cannot be interpreted. As a result, the performance of an IVDMIA test "involves steps ... that are not within the ordinary "expertise and ability" ( $p$. 2) of laboratories working in the area of LDTs.

It is worth citing the complete definition of IVDMIAs used in the First Draft Guidance:

For purposes of this guidance, IVDMIAs are test systems that employ data, derived in part from one or more in vitro assays, and an algorithm that usually, but not necessarily, runs on software to generate a result that diagnoses a disease or condition or is used in the cure, mitigation, treatment, or prevention of disease. IVDMIAs reflect the following characteristics:

1. Use clinical data ... to empirically identify variables and to derive weights or coefficients employed in an algorithm;

2. Employ the algorithm to integrate these variables in order to calculate a patient-specific result ... . This result cannot be independently derived and confirmed by another laboratory without access to the proprietary information used in the development and derivation of the test; and

3. Report this result, which cannot be interpreted by the well-trained health care practitioner using prior knowledge of medicine without information from the test developer regarding its clinical performance and effectiveness.

A clear indication of the emergent nature of the entities it designated, this seemingly clear-cut definition quickly generated a fair amount of discussion. To borrow from the vocabulary of actornetwork theory, the First Draft Guidance inaugurated a process of problematization, whereby the FDA attempted to define a new hypothetical reality, introduce a new project, identify new stakeholders and turn the resulting entities into "matters of concern" (Callon, 1986; Callon \& Rabeharisoa, 2008). Faced with stiff criticism, the FDA soon admitted that its problematization of IVDMIAs was deficient and asked meeting participants for "concrete suggestions on how to make the definition ... clearer" (FDA Meeting, p. 9). A number of participants (including those who supported the new regulation) had previously faulted the definition for its insistence on the presence of an algorithm. FDA officials quickly conceded that not all tests relying on an algorithm were necessarily IVDMIAs; even multivariate tests that simultaneously measure several parameters did not necessarily qualify as IVDMIAs. As a result, the Second Draft Guidance focused on the lack of transparency to clinical end users rather than the presence of an algorithm per se:

An IVDMIA is a device that:

1) Combines the values of multiple variables using an interpretation function to yield a single, patient-specific result ... that is intended for use in the diagnosis of disease or other conditions, or in the cure, mitigation, treatment or prevention of disease, and

2) Provides a result whose derivation is non-transparent and cannot be independently derived or verified by the end user. 
The claim that the results of the tests cannot be independently verified underscores their bioclinical nature, i.e., the fact that the knowledge and skills necessary for a correct interpretation of test results are distributed among different actors in different spaces, namely biologists, bioinformaticians and biostatisticians, the laboratories and companies producing the tests, and the clinicians who use them. The Guidance took pains to highlight this aspect of the new definition:

While the input variables, alone or in combination, might have meaning to the clinician, the clinician could not verify the clinical significance of the IVDMIA result on his or her own. In addition, the ordering physician cannot reach the IVDMIA result on his or her own, nor could he or she independently interpret that result. The ordering clinician requires information from the test developer, rather than generally accepted information from the clinical community, in order to interpret the IVDMIA result for use in the management of the patient.

But if this is so, not only does the complex multivariate analysis lie beyond the skills of the "well-trained" clinician, the results themselves convey a clinical interpretation insofar as they assign the patient to a specific clinical category - e.g. low risk vs. high risk - that in turn directs therapy. While clinicians maintain that they can use this information to fine-tune therapy, thus exercising their clinical autonomy (interview with a Dutch oncologist, 23 June 2010), the result of the test is not merely a biological value whose clinical meaning requires triangulation with the outcome of clinical examinations: the clinical interpretation lies within the IVDMIA. True, clinicians may ignore discordant results and rely instead on their clinical expertise and judgment. But can they "tweak" the results to make sense of an apparent anomaly? And what if, as recalled by a French clinician (interview, 21 April 2008) a patient, faced with different treatment options (chemotherapy or not) requests a test as a way of obtaining a second (non-human) opinion in addition to her treating physician's recommendation? Last but not least, what about the pathologists who not only see their traditional techniques challenged by the new molecular tools but must now bear witness to the emergence of proprietary tests that escape their immediate control? In what follows we will focus on these issues that, taken together, signal a redefinition (albeit disputed) of the domain of diagnosis and a concurrent shift in the locus of clinical judgment and clinical decision-making.

\section{Biological tests or clinical procedures? Displacing clinical judgment and clinical decision- making}

The questions that closed the previous section bring us to the core of our subject matter. If, as claimed by the Second Draft Guidance, clinicians are unable to "verify the clinical significance of the IVDMIA result", and if the "ordering physician [cannot] reach the IVDMIA result on his or her own, nor [can] he or she independently interpret that result", the genomic test embodies (non-human) clinical-diagnostic agency. As a result, the evidentiary basis for clinical judgment no longer lies in the "generally accepted information from the clinical community" but, rather, in the test itself and thus out of the clinician's purview. Not only can an IVDMIA report qualify as a full-fledged medical diagnosis rather than a mere biological result, but such a report, by including prognostic and predictive information, also dictates therapeutic decisions more stringently than traditional diagnosis. At a recent breast cancer meeting, for instance, a heated

discussion opposed clinicians who suggested that genomic biomarkers should replace traditional 
clinico-pathological parameters, such as the degree of spread of a tumor, thus reducing a treating physician's therapeutic leeway (fieldnotes, EBCC-7 Meeting, Barcelona 2010).

During the IVDMIA debate, FDA opponents questioned whether these tests could indeed be construed as a clinical diagnosis rather than a biological service and, in particular, disputed their characterization as black boxes whose content clinicians were unable to access. A spokesperson for a molecular diagnostic company, for instance, rejected the claim that physicians were unable to interpret and assess test results, arguing that their very training prepared them to juggle "multiple kinds of data, including clinical history, signs and symptoms, and test results, in counseling patients" (Dockets EC47, p. 2). Another company's representative argued that "in every case, it is the treating physician that makes the choice about which validated test is appropriate for a particular patient and to ensure that each test is medically necessary" (FD $A$ Meeting, p. 102). According to yet another speaker the test and its application "[did] not stand alone in clinical decision making" and were appraised in conjunction with additional clinical and laboratory information (Ibid., pp. 182-3). The clinicians' domain of expertise, under this description, is more than likely to expand, enabling them to easily incorporate the results provided by IVDMIAs into the overall diagnostic picture. Along similar lines, the Coalition for $21^{\text {st }}$ Century Medicine (Dockets EC38, p. 5) chastised the FDA's "inherently subjective assessment of what a well-trained healthcare practitioner can or cannot interpret", arguing that "once a sufficient number of practitioners [had] become familiar with the assay measurement", a test initially categorized as IVDMIA under the FDA definition would no longer qualify as such.

The debate thus confronted two opposing interpretations of current diagnostic trends. One camp saw genomic tests (and the companies that produce them) as potentially trespassing on the clinical domain and interfering with clinical judgment; the other stressed the capacity of clinical skills to assimilate the new genomic tools by adjusting the content of clinical expertise without reducing its import. Commenting on his routine use of tumor profiling tests, a California breast cancer specialist asserted: "That's how I got from being an empiricist to being more precise, maybe a 'precisionist', in the way I handle patients" (cited in Garber, 2011, p. 86). Genomic tests, in his view, enlarge a clinician's diagnostic capacity. What the FDA construed as a structural impossibility (clinicians cannot interpret the test because they don't have access to the necessary clinical information) was recast as a matter of learning new skills and as an opportunity to extend them. These opposing interpretations have their counterpart in sociological debates about whether (and to what extent) technology "deskills" practitioners or whether, as argued for instance by Hartland (1993) in her case study of electrocardiography, rather than eliminating the clinicians' quintessential tacit knowledge, new technologies simply shift it to novel domains.

Attempts to capture or frame complex situations via generic, abstract categories such as "Technology" often lead nowhere; attention to the details of specific technologies are more fruitful. This lesson was not lost on those FDA Meeting speakers who argued that not all IVDMIAs were alike with regards to clinical interpretation. A representative of the American Clinical Laboratory Association, for instance, requested that the FDA limit its definition of IVDMIAs to tests that "generate a patient specific binary result that is intended definitively to diagnose a condition or to direct behavior for the cure, mitigation, treatment or prevention of disease" (p. 75). Similarly, the speaker for the Coalition for $21^{\text {st }}$ Century Medicine noted that the level of risk should be a key consideration, and that risk is "higher for IVDMIAs that are predictive and that result in a binary therapy recommendation to treat or not to treat based solely 
on the IVDMIA outcome"; lower-risk tests that "do not give binary therapy recommendations" should not be submitted to the same regulatory strictures (pp. 50-51). This approach corresponds, in part, to the two different strategies pursued by the makers of Oncotype DX and MammaPrint. The latter, as we saw, provides a binary result, whereas the former offers a numerical score that, according to its developers, adapts the test to existing clinical decision-making routines. Faced with clinicians who are interested in the expression scores for a small subset of genes with which they are already familiar, the company's representatives stress that their test results include, in addition to the RS score, individual values for those genes (fieldnotes, EBCC-7, Barcelona, 24-27 March 2010). The makers of Oncotype DX, in other words, inoculated their test against possible criticism, incorporating variables designed to overcome the accusation of encroaching upon clinicians' privileges. Unsurprisingly, the spokesperson for MammaPrint rejected this pretension, insisting that the "complexity of IVDMIA test systems ... warrants oversight because experimental design of validation studies is not straightforward ... Algorithms employed require independent review and validation by the FDA. Otherwise they are just a black box" (FDA Meeting, p. 207).

The discussion took an even more explicit "sociological" turn when participants moved from "in principle" arguments about clinical expertise to an "in practice" assessment of the clinicians' skills and range of options. At the FDA Meeting a spokesperson for the American Society for Microbiology argued that the biomedical literature and direct-to-clinician marketing put clinicians under increased pressure to use molecular tests. Yet, these tests "bypassed the expertise of anyone who can objectively look at the quality of the information being provided". As a result "clinicians despite their skill and their excellent knowledge of clinical diseases are not equipped to know whether the test is one with which they should be managing their patients" (pp. 65-6). While the previous speaker cited her own experience as evidence, a representative of the Genetics and Public Policy Center cited a 2006 survey of 190 laboratory directors, showing that 36\% of the polled directors "lack[ed] a clear understanding of what clinical validity means" (p. 108). Breast cancer patient advocates echoed similar sentiments, noting that unless the FDA decided to regulate IVDMIAs, their commercialization would be left to the companies that write the best press releases, do the most advertising, or try and court advocacy groups (p. 128). The FDA should therefore expand the scope of its review to include proof that use of biomarkers would result in better patient outcomes (p. 201) or, in other words, not only clinical validity but also clinical utility (p. 205). Indeed, we can fairly ask: what is the clinical utility of these tests or, in simpler words, what kind of information do they provide?

\section{Prognosis and prediction: regulating drugs, regulating tests}

As we saw, the new genomic tests are not "mere" diagnostic tests; they provide prognostic and predictive information. The FDA distinguished between prognosis and prediction to assign tests to different risk-based regulatory categories: "a device intended as an indicator of a patient's risk of cancer recurrence may be a class II device [...] while the same device intended to predict which patients should receive chemotherapy might require premarket approval [a class III device]" (Second Draft Guidance, p. 8). Traditionally, the terms prediction and prognosis have often been used interchangeably: to make a prognosis means to predict what will happen to the patient and her tumor. Prognostic assessments, in turn, provide a basis on which to decide about the most appropriate treatment (e.g., chemotherapy for high-risk but not for low-risk patients). 
The tests discussed in this article contribute to a growing disjuncture between prognosis and prediction: the former now refers to the disease itself (how will it evolve) whereas the latter refers to how a patient will respond to a given treatment, either in a broad sense, e.g. "chemotherapy", or, more decisively, in relation to a specific drug as part of the promissory program of "personalized medicine". The prognostic/predictive distinction is widely reflected in debates and controversies over the different clinical and statistical procedures used to validate prognostic vs. predictive claims, over the extent to which the prognostic and/or predictive properties of a test contribute to its clinical utility, and to disputes about the comparative value of competing commercial assays such as the Oncotype DX and MammaPrint tests (Kohli-Laven et al., 2011). Conference sessions and even entire meetings (e.g., the Conference on "Prognosis and Prediction in Breast Cancer" held in Monaco in October 2008) have been devoted to this thorny issue. Participants themselves have often expressed perplexity. At the FDA Meeting, for instance, an internist turned lawyer noted that "it's not clear what is the difference between a prognostic claim and a predictive claim. Generally, you will have a claim that is talking about the likelihood of some outcome, survival, disease free survival, cancer recurrence, a cardiac event. That's going to be on some therapy. Is that a prognostic claim or is that a predictive claim with respect to the particular therapy?" (pp. 16-7).

In spite of the confusion surrounding it, the prognosis/prediction distinction figures prominently in ongoing debates. At the FDA Meeting, a pathology professor noted that present-day pathology reports "go well beyond the diagnosis found on the top line to include items found in the middle on prognosis and targets of therapy" (p. 24). Another pathologist described a test as "the first diagnostic test in pathology that determines whether or not a patient will be treated with a specific therapy irrespective of any clinical input" (p. 60; our emphasis). Once again, we face the issue of clinical judgment. During the aforementioned Monaco meeting on "Prognosis and Prediction in Breast Cancer" the medical director for an IVDMIA company told one of us that the shift from prognostic to predictive amounted to a "paradigm shift": while oncologists often spoke of prognosis and selected therapy based on prognosis, the new tests allowed them to select therapy directly on predictions (fieldnotes, October 2008). In fact, the new diagnostic tests open up an unexplored domain of interventions not only for oncologists, but also for industry - insofar as drug companies can create platforms for establishing the value of a compound independently from prognostic criteria-and for patients, who can use a test to double-check the therapeutic recommendation of their treating physician, and thus work around clinical expertise (therefore also raising the issue of patients' access to their tumor specimens; Ploem et al., 2010).

The debate concerning clinical judgment does not rest on a simplistic opposition between the clinic and the biology laboratory. The clinical side of the equation encompasses a variety of actors, including not only the treating physicians (medical oncologists and/or surgeons) but also pathologists. As both physicians and gatekeepers of laboratory medicine, pathologists via their powerful professional organization - the College of American Pathologists (CAP) - are involved in the accreditation of clinical laboratories. Moreover, pathologists have traditionally provided diagnostic and prognostic information to treating physicians on the basis of their extensive visual skills in examining tissue slides. Genomic signatures, thus, are of particular relevance for their work. As noted in a clinical pathology journal:

Not surprisingly, apart from a few exceptions, most pathologists have been reluctant to deal with this new technology and feel that their role in "guiding the surgeon's hand" would be 
in jeopardy. This has been aggravated by the attitude of some scientists and clinicians, who have deemed current pathology methods as unsophisticated and obsolete, and compared them to some ritualistic practices of primitive tribes (Reis-Filho, Westbury, \& Pierga, 2006, p. 225).

The polemical tone of the preceding quote resonates with more recent exchanges, during which pathologists promoting or dismissing the added value of molecular profiling have crossed swords (Shiang \& Pusztai, 2010; Weigelt \& Reis-Filho, 2010). CAP's reaction to the IVDMIA initiative has been guarded: while recognizing that "complex diagnostic tests are being developed and made available that are not subject to traditional peer reviews and independent verification", they also argued that the enhancement of CLIA to include demonstration of clinical validity could solve the problem since this was the road already taken by the College's own laboratory accreditation program (Dockets, C4). A subsequent document attributed the role of intermediary between the test and the treating physician to the pathologist: "due to the complexity of IVDMIAs, pathologists and clinical geneticists are likely to be asked by clinicians to help interpret the results obtained from these assays as they relate to patient management" (CAP, 2009 , p. 2). Yet, in spite of the fact that pathologists are "in the ideal position to assimilate the results of [IVDMIAs] with other laboratory and clinical information for a given patient", the tests could be "potentially problematic" for them "since closed algorithms do not allow pathologists to easily understand or interpret how the IVDMIA result is obtained" (CAP, 2009, p. 3). In yet another subtle shift towards the FDA position, at a 2010 molecular pathology meeting a CAP representative commented on the proposed LDT regulation arguing that while the FDA "should continue to exercise enforcement discretion in regulating LDTs", it should "assert authority" on high-risk LDTs, i.e. those whose predictive nature leads to therapeutic decisions (fieldnotes, Association for Molecular Pathology 2010 Annual Meeting, San José, CA).

These cautious statements translate the fluidity of the present situation. On the one hand, pathologists, via their professional spokespersons, tend to assert their traditional role as intermediaries between tests and physicians. On the other hand, genomic tests challenge such a role. At a 2008 meeting a pathologist showed a cartoon of a line-up of pathologists at the unemployment office, before quickly reassuring her colleagues that pathologists were likely to remain employed for any foreseeable future (fieldnotes, Monaco Meeting, October 2008). More significantly, the aforementioned exchange about the value of genomic tests constitutes evidence of the emergence of fault lines at the core of cancer pathology practices. The issue is not that pathologists will lose their jobs or that biology will somehow lead to the death of the clinic. Rather, the ascription of diagnostic, and in particular predictive agency to genomic tests turns them into key non-human intermediaries between medical oncologists, pathologists, medical biologists, and their patients, and in so doing rearranges the evidentiary basis that generates public trust in medical practices.

While prognostic/predictive tests that examine gene expression profiles should not be confused with "companion tests" for targeted drugs - i.e., tests that establish whether a patient's tumor expresses a particular receptor targeted by the drug (Taube et al., 2009) - there are obvious parallels between them. A clinical researcher involved in the design of clinical trials for targeted therapies noted that while regulatory frameworks and regulatory cultures for tests and drugs were quite different, the situation has evolved: 
So a diagnostic test is considered a device in the usual ... FDA model. In a way it's very similar to a scalpel, ... basically it's supposed to do what it is supposed to do [and] it should be safe. ... A diagnostic test in a regulatory framework is like that. It should do what you claim it does ... and do it accurately. ... Now, we are using diagnostic tests to make treatment decisions. It's not a device anymore; it's more like it falls into a therapeutic model. ... The reason the FDA needs to put these together is because ... it's one of the only organizations that have these two cultures embedded within individuals within the same building (interview, 30 September 2010).

Sociologically speaking, the issue is trust in the tools of contemporary biomedicine. Not all prescribing physicians are aware of the intricacies of the clinical trials that are required to prove a new drug's safety and efficacy, but once the latter has been established by clinical research and sanctioned by the FDA, practicing physicians are expected to incorporate the drug into their treatment plans, making adjustments when necessary. Clinicians can trust (with some caution) drugs precisely because their clinical peers have clinically validated them following a series of steps prescribed by the FDA. Clinical judgment, therefore, simultaneously mobilizes a situated expertise in managing a patient and an element of trust in the evidence that underpins the clinical validity of the substances used in routine practices. Clearly on display in the case of drugs, the presence of such expertise and trust is a matter of concern in the case of diagnostic procedures: as we saw in the case of MammaPrint and Oncotype DX, while makers of the former chose to go the regulatory route that mimics the approval of drugs, producers of the latter opted for a professional network outside the FDA control.

By shifting the content, contours and tools of diagnosis, and by establishing a direct connection between test results and therapeutic choices, genomic tests extend the essential tension between situated clinical expertise and trust in the clinical community that characterizes prescription drugs to the diagnostic domain. Companies are now working to "develop tests that will determine which specific therapies may work best for a specific patient", thus moving "beyond tests that can provide 'yes/no' responses to whether a chemotherapy ... or a hormonal therapy ... may work" (Fong, 2011). According to the head of a diagnostic company, it would be unthinkable to trust a drug simply because a colleague or a group of expert colleagues say that it is good based on their assessment; given the link to therapy, standards similar to those required from drugs should apply to diagnostic products (fieldnotes, June 2010). As argued by a panelist from the Walter Reed Army Institute of Research at the LDT Meeting (pp. 175-6) "many clinicians ... would like to have some sort of third party validation ... a peer review ... an FDA qualification process or some other qualification process that would give you that peer review", a sentiment echoed by the President of the National Society of Genetic Counselors, according to whom "in general and across specialties, clinicians trust the analytical results of LDTs, and state and Federal regulation should ensure that this trust is well placed" (pp. 111-2).

We began this paper by examining the debate about the extent to which genomic tests impinge upon clinical jurisdiction. Following that debate has led us to the issue of the evidentiary basis of medicine as part of a redefinition of the relations between diagnosis and therapy. These two elements are not distinct; rather, in this post-genomic era, they are the two sides of the same coin.

\section{Conclusion}


Our analysis raised the question of the extent to which genomic tests participate in the realignment of clinical expertise and biological information, in particular with regards to the expansion of the domain of diagnosis to include new kinds of prognostic and predictive information. We have done so by examining how the actors themselves discuss and debate this issue and its ramifications as part of the process of problematization inaugurated by the FDA. As argued elsewhere (Bourret, 2005; Cambrosio et al., 2006; Rabeharisoa \& Bourret, 2009), the objective of regulation in state-of-the-art domains such as genomics is not simply the stabilization of the new tools from a technical point of view, but to define the entities that make up those tools as well as the frameworks for their implementation. IVDMIAs cannot be conceived of as autonomous technological forces insofar as they cannot be extracted from the sociotechnical networks in which they are embedded. While the FDA regulatory initiative can be said to contribute to the production of new entities (and not simply in a nominalist sense), these new entities have in turn led to changes in regulation. Nor can IVDMIAs be reduced to the social contingencies that underlie their constitution as a regulatory category, since, in addition to their obvious material dimension, one of the outcomes of their implementation is the disruption of existing social arrangements, and in particular the existing configuration of medical work, by redistributing roles and agencies among an evolving set of actants. One of the possible consequences of such redistribution is a shift in the locus of clinical judgment and clinical decision-making. Whether this will be the case, as some of the actors seem to believe, and if so to what extent; whether clinicians will continue to rely on more traditional pathology parameters to inform their judgment, as others maintain; or whether and how clinicians will manage to integrate the new tools into their set of clinical skills, as yet others argue, remains an open question. For our present purpose, the important fact is that this question has become an ongoing concern, and that scenarios corresponding to these different possibilities are built into the molecular diagnostic tools.

\section{References}

Alizadeh, A.A., Eisen, M.B., Davis, R.E., Ma, C., Lossos, I.S. et al. (2000). Distinct types of diffuse large B-cell lymphoma identified by gene expression profiling. Nature, 403, 503-511.

Berg, M. (1992). The construction of medical disposals, medical sociology and medical problem solving in clinical practice. Sociology of Health and Illness, 14, 151-180.

Boltanski, L. \& Thévenot, L. (1999). The sociology of critical capacity. European Journal of Social Theory, 2, 359-477.

Boltanski, L. \& Thévenot, L. (2006). On justification: economies of worth. Princeton, NJ: Princeton University Press.

Bourret, P. (2005). BRCA patients and clinical collectives: new configurations of action in cancer genetics practices. Social Studies of Science, 35, 41-68. 
Callon, M. (1986). Some elements of a sociology of translation: the domestication of the scallops and the fishermen of Saint Brieuc Bay. In J. Law (Ed.), Power, action and belief: a new sociology of knowledge? (pp. 196-229). London: Routledge \& Kegan Paul.

Callon, M. \& Rabeharisoa, V. (2008). The growing engagement of emergent concerned groups in political and economic life: lessons from the French association of neuromuscular disease patients. Science, Technology \& Human Values, 33, 230-261.

Cambrosio, A., Keating, P., Schlich, T., \& Weisz, G. (2006). Regulatory objectivity and the generation and management of evidence in medicine. Social Science \& Medicine, 63, 189-199.

CAP (2009). College of American Pathologists Technology Assessment Committee, In vitro diagnostic multivariate index assays (IVDMIA), available at: http://www.cap.org/apps/docs/committees/technology/ivdmia.pdf

Christakis, N. (1999). Death foretold: prophecy and prognosis in medical care. Chicago, IL: University of Chicago Press.

Evans, B.J. (2006). What will it take to reap the clinical benefits of pharmacogenomics? Food and Drug Law Journal, 61, 753-794.

Fong, T. (2011) Agendia Planning to File IPO in 2011 Genome Web, January 14; available at: http://www.genomeweb.com/print/959748?hq_e=el\&hq_m=908479\&hq_l=1\&hq_v=c4e8b24 29

Garber, K. (2011). Ready or not: personal tumor profiling tests take off. JNCI, 103, 84-86.

Gibbs, J.N. (2007). ASRs and IVDMIAs: FDA's new draft guidances. Two new draft guidances could affect how laboratory tests are developed in the future. IVD Technology, available at http://www.devicelink.com/ivdt/archive/07/03/012.html

Hartland, J. (1993). The use of "intelligent" machines for electrocardiograph interpretation. In G. Button (Ed.), Technology in working order: studies of work, interaction, and technology (pp. 55-80). London: Routledge.

Jutel, A. (2009). Sociology of diagnosis: a preliminary review. Sociology of Health \& Illness, 31, 278-299.

Keating, P. \& Cambrosio, A. (2003). Biomedical platforms. Realigning the normal and the pathological in late-twentieth-century medicine. Cambridge, MA: MIT Press.

Keating, P. \& Cambrosio, A. (2004). Does biomedicine entail the successful reduction of pathology to biology? Perspectives in Biology and Medicine, 47, 357-371.

Knaapen, L., Cazeneuve, H., Cambrosio, A., Castel" P. \& Fervers, B. (2010). Pragmatic Evidence and Textual Arrangements: A Case Study of French Clinical Cancer Guidelines. Social Science \& Medicine, 71, 685-692. 
Kohli-Laven, N., Bourret, P., Keating, P., \& Cambrosio, A. (2011) Cancer clinical trials in the era of genomic signatures: Biomedical innovation, clinical utility, and regulatory-scientific hybrids. Social Studies of Science (in press).

Lakhman, K. (2006). OIVD wants to discuss Oncotype Dx's status with Genomic Health; more insight into FDA's IVD goal? Pharmacogenomics Reporter (February 1), available at http://www.genomeweb.com/print/672820.

Latour, B. (2005). Reassembling the social: an introduction to actor-network-theory. Oxford: Oxford University Press

Mol, A. (2002). The body multiple. Ontology in medical practice. Durham: Duke University Press.

Moreira, T. (2005). Diversity in clinical guidelines: the role of repertoires of evaluation. Social Science \& Medicine, 60, 1975-1985.

Nachi, M. (2006). Introduction à la sociologie pragmatique. Paris: Armand Colin.

Perou, C.M., Sørlie, T., Eisen, M.B., van de Rijn, M., Jeffrey, S.S., et al., (2000). Molecular portraits of human breast tumours. Nature, 406, 747-752.

Ploem M.C., Retèl, V.P., Linn S.C., van Boven, H.H., Schmidt, M.K. et al. (2010). Tumour tissue: who is in control? Lancet Oncology, 11, 9-11.

Q\&A: FDA's Zineh reviews 2010 personalized Rx efforts and discusses future plans (2011), Genome Web, January 5, available at:

http://www.genomeweb.com/print/958917?hq_e=el\&hq_m=903163\&hq_1=1\&hq_v=c4e8b24 249

Rabeharisoa, V., \& Bourret, P. (2009). Staging and weighting evidence in biomedicine: comparing clinical practices in cancer genetics and psychiatric genetics. Social Studies of Science, 39, 691-715.

Reis-Filho, J.S., Westbury C., \& Pierga, J.-Y. (2006). The impact of expression profiling on prognostic and predictive testing in breast cancer. Journal of Clinical Pathology, 59, 225-231.

Shiang, C. \& Pusztai, L. (2010). Molecular profiling contributes more than routine histology and immonohistochemistry to breast cancer diagnostics. Breast Cancer Research, 12(Suppl 4), S6.

Taube, S.E., Clark, G.M., Dancey, J.E., McShane, L.M., SIgman, C.S., \& Gutman, S.I. (2009). A perspective on challenges and issues in biomarker development and drug and biomarker codevelopment. JNCI, 101, 1453-63.

Weigelt, B. \& Reis-Filho, J.S. (2010). Molecular profiling currently offers no more than tumour morphology and basic immunohistochemistry, Breast Cancer Research, 12(Suppl 4), S5. 
Woodcock, J. (2007). The prospects for "personalized medicine" in drug development and drug therapy. Clinical Pharmacology and Therapeutics, 81, 164-169. 